\title{
Microfluidic Chain Reaction
}

Mohamed Yafia, ${ }^{1,2, \dagger}$ Oriol Ymbern, ${ }^{1,2, \dagger}$ Ayokunle O. Olanrewaju ${ }^{1,2, \S}$, Azim Parandakh ${ }^{1,2}$, Ahmad $^{2}$ Sohrabi Kashani ${ }^{1,2}$, Johan Renault ${ }^{1,2}$, Zijie Jin ${ }^{1,2}$, Geunyong Kim ${ }^{1,2}$, Andy $\mathrm{Ng}^{1,2}$, David Juncker ${ }^{1,2, *}$

1. Biomedical Engineering Department, McGill University, Montreal, QC, Canada

2. McGill Genome Centre, McGill University, Montreal, QC, Canada

${ }^{\dagger}$ Equal contribution. ${ }^{\S}$ Current address: Mechanical Engineering Department, University of Washington, Seattle, WA, USA.

\section{Abstract:}

Chain reactions are characterized by initiation, propagation and termination, are stochastic at microscopic scales and underlie vital chemical (e.g. combustion engines), nuclear and biotechnological (e.g. polymerase chain reaction) applications. ${ }^{1-5}$ At macroscopic scales, chain reactions are deterministic and limited to applications for entertainment and art such as falling domino and Rube Goldberg machines. Appositely, microfluidic lab-on-a-chip (also called a micro total analysis system), ${ }^{6,7}$ which were envisioned pursuant to microelectronic integrated circuits, are generally not integrated on a chip owing to an enduring dependency on cumbersome connections, peripherals, and on computers for automation. ${ }^{8-11}$ Capillary microfluidics integrate energy supply and flow control onto a single chip by using capillary phenomena, but programmability remains rudimentary with at most a handful (eight) operations possible. ${ }^{12-19}$ Here we introduce mesoscopic microfluidic chain reactions (MCRs) based on capillary phenomena for reliable programming and automation of complex liquid handling algorithms integrated in a chip. MCRs are encoded into the chip microarchitecture, 3D printed as a monolithic circuit, and deterministically propagated by the free-energy of a paper pump. With MCR, we sequentially triggered the release of 300 aliquots across chained, interconnected chips, and automated a protocol for SARS-CoV-2 antibodies detection in saliva with visual and quantitative results by cell phone imaging. We automated and miniaturized for the first time the labor-intensive thrombogram with serial and parallel operations including timers and iterative cycles of synchronous flow and stop-flow sequences. Thrombograms with normal, hemophilialike, and anticoagulant-spiked plasma were generated. MCRs are generalizable, and both the density and number of chain reaction units are scalable. MCRs are untethered from and unencumbered by peripherals, encode programs structurally in situ, and form a frugal, versatile, 
bona fide lab-on-a-chip with wide-ranging applications in liquid handling and point-of-care diagnostics.

\section{Results:}

MCR encode the deterministic release of reagents stored in a series of reservoirs, with the release of reservoir $n$ being conditional on emptying (draining) of the reagent in reservoir $n-1$, and emptying reservoir $n$ in turn triggering the release of reservoir $n+1$. Capillary domino valves (CDVs) encode this condition, and serially connect, i.e. chain, the reservoirs, and thus control the propagation of the chain reaction ${ }^{1}$ (Fig. 1a). MCRs were implemented in 3D-printed circuits using a common stereolithography printer with feature size from $100 \mu \mathrm{m}$ to $1.5 \mathrm{~mm}$, hydrophilized using a plasma (see Supplementary Fig. S1 and S2), sealed with a plain cover, and connected to a capillary pump made of paper (filter papers or absorbent pads). The paper is spontaneously wetted by aqueous solution drawn from the microfluidic circuit by releasing free energy stored in the paper surface, and drives the chain reaction; expressed differently, the capillary pump generates a negative capillary pressure that is hydraulically propagated back into the circuit via the main channel and serially drains side-reservoirs connected via a small conduit, called functional connection (further described below). CDVs form air links between adjacent reservoirs, serially connecting them along a path parallel to the main channel, but with filled reservoirs forming a liquid plug between CDV air links. When each reservoir empties, the length to the continuous air link increases from an air vent up to the next filled reservoir in the MCR, (Fig. 1a-d, and Video S1). This simple design structurally encodes the sequential release of an arbitrary number $\mathrm{N}$ reservoirs without peripheral connection or moving parts.

MCR require ancillary capillary microfluidic components that fulfill different functions depending on the intended operation (e.g. loading, holding, mixing, and draining liquids following the MCR progression) to form fully integrated and scalable capillaric circuit (CCs). CCs are designed based on a library of building blocks including capillary pumps, flow resistances, and many types of capillary valves (stop v., trigger v., retention v., retention burst v.) ${ }^{13,14}$, and thus analogous to microelectronic integrated circuits, but lacking scalability and functionality theretofore. In MCRs, samples are loaded by capillary flow via an inlet with a capillary retention valve and entirely fill the reservoirs lined with three stop valves, including

\footnotetext{
${ }^{1}$ In an earlier implementation of CDVs and air links were made in a hydrophobic cover material that was aligned and sealed on top of the capillaric circuit designed in a hydrophilic base (See Supplementary Fig. S3).
} 
two with a dual retention burst valve function connecting to the two lateral CDVs, and one at the intersection of the functional connection and the main channel (Fig. 1c). Although the functional connection is a deceptively simple straight channel, it fulfills five key functions. It is (i) the air vent during filling of the reservoir, and (ii) a stop valve preventing the reagent from spilling into the main channel while it is empty. After filling of the main channel, it forms a (iii) hydraulic link propagating the pressure from the main channel into the reservoir. (iv) It becomes the outlet and a flow resistance (discussed further below) during reservoir emptying, and (v) a capillary retention valve stopping air from invading the main conduit after the reservoir is emptied. As a result, many trade-offs guide its design.
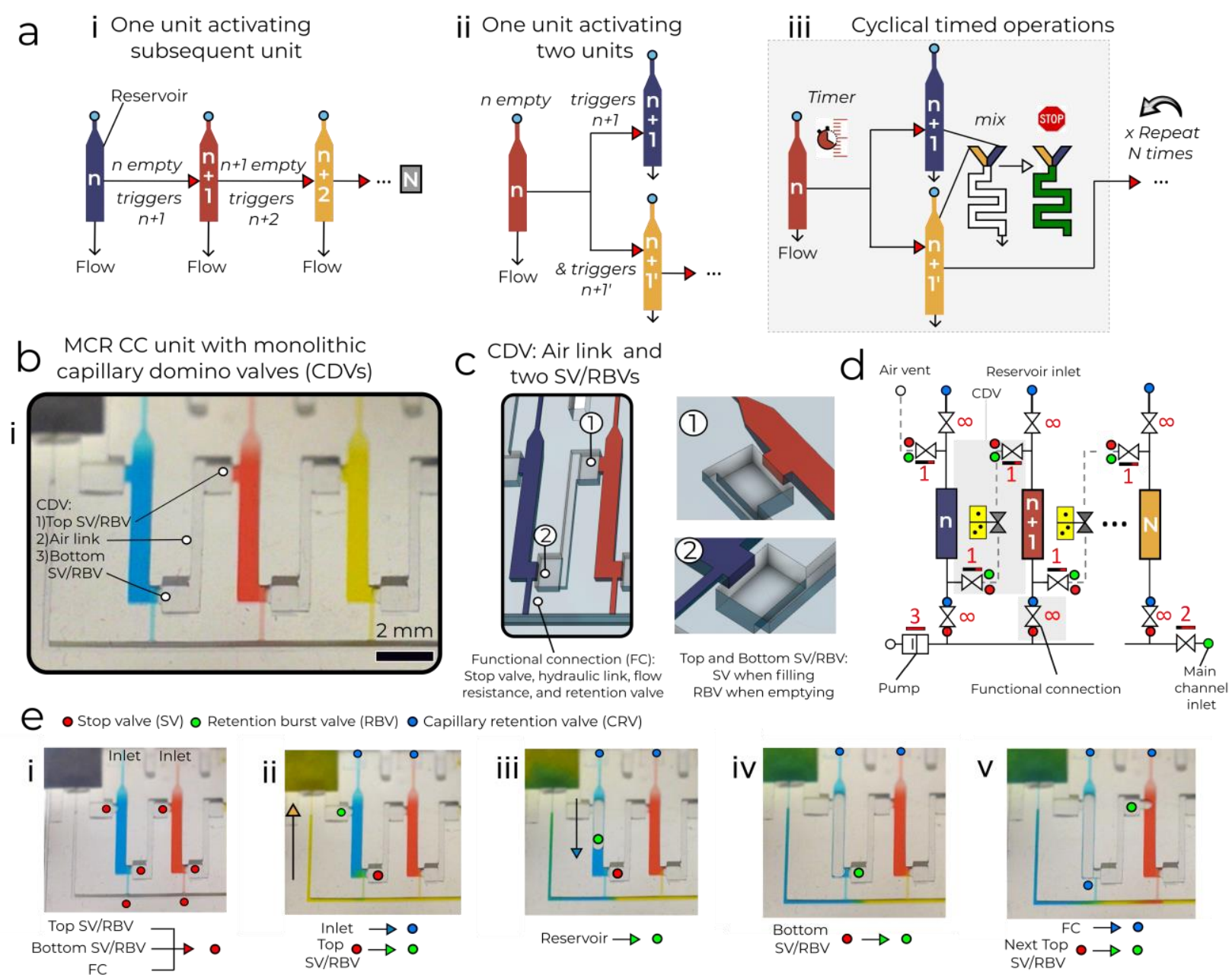

Fig. 1. Microfluidic chain reaction (MCR) with monolithic capillary domino valve (CDV) for serial delivery of reagents in capillaric circuits (CCs). (a) i- Serial MCR, ii- Branching MCR, iii- Cascaded, timed MCR. (b) MCR unit with close-up of the reservoirs chained via 
CDVs. (c) Close-up of CDV design: Air links form a pneumatic connection and capillary stop valves keep liquids out. (d) Symbolic view of the MCR unit with CDV (grey overlay) that includes an air link, two stop valves (SVs) and retention burst valves (RBVs). (e) Screen shots of Video S1 showing sequential steps for MCR where most of the capillary elements have dual functions that change upon either loading or during MCR. i- A loaded chip where the liquids stop filling at different locations. ii- MCR is triggered (the inlet becomes a capillary retention valve and the top SV becomes a RBV). iii- Emptying of the first reservoir upon bursting of the top RBV. iv- The bottom SV momentarily becomes an RBV that bursts immediately. v- Air now occupies the emptied reservoir. The functional connection (FC) becomes a retention valve preventing the air from penetrating into in the main channel. The air link connects the air to the RBV of the next reservoir, which bursts, and triggers reservoir emptying.

We sought to understand the design window and failure modes of MCR, notably under which conditions downstream of CDVs might trigger prematurely, using both theory and experiments. We analyzed the MCR based on an electrical analogy. ${ }^{20}$ Successful and incremental propagation of the MCR is conditional on preventing the breach of the liquid in reservoir $n$ into the CDV and air link connecting $n+1$, which is equivalent to stating that all the liquid must flow exclusively through the functional connection. This condition is satisfied when (see supplementary information for detailed derivation):

$$
P_{F C(n)}<P_{B U R S(n)}+P_{R B V(n+1) \quad \text { Equation } 1}
$$
with $P_{B U R S}$ being the leakage pressure of the stop valve, $P_{R B V}$ the retention pressure of the retention burst valve at the extremities of the air link (and CDV) connecting reservoir $n$ and $n+1$, respectively. We then derived $P_{B U R S}$ (numerically) and $P_{R B V}$ (analytically) for the $3 \mathrm{D}$ printed $\mathrm{CCs}$, and measured them for confirmation, Fig. 2b. Next, several MCRs featuring functional connections with large, and increasing $R_{F C}$ were tested with pumps with different capillary pressure and flow rates. The interplay between the resistance and the flow rate determines the operational window for the CDV while they are inversely proportional. We found excellent concordance between theory and experiments for the operation window of the MCR, and failure only occurred for the highest values of $R_{F C}$ (no. 5 and 6), and only for the most powerful capillary pumps, Fig. 2c,d. The MCR designs used in the applications shown below, are well within the failure threshold and guarantee the reliability and robustness of the MCR. 

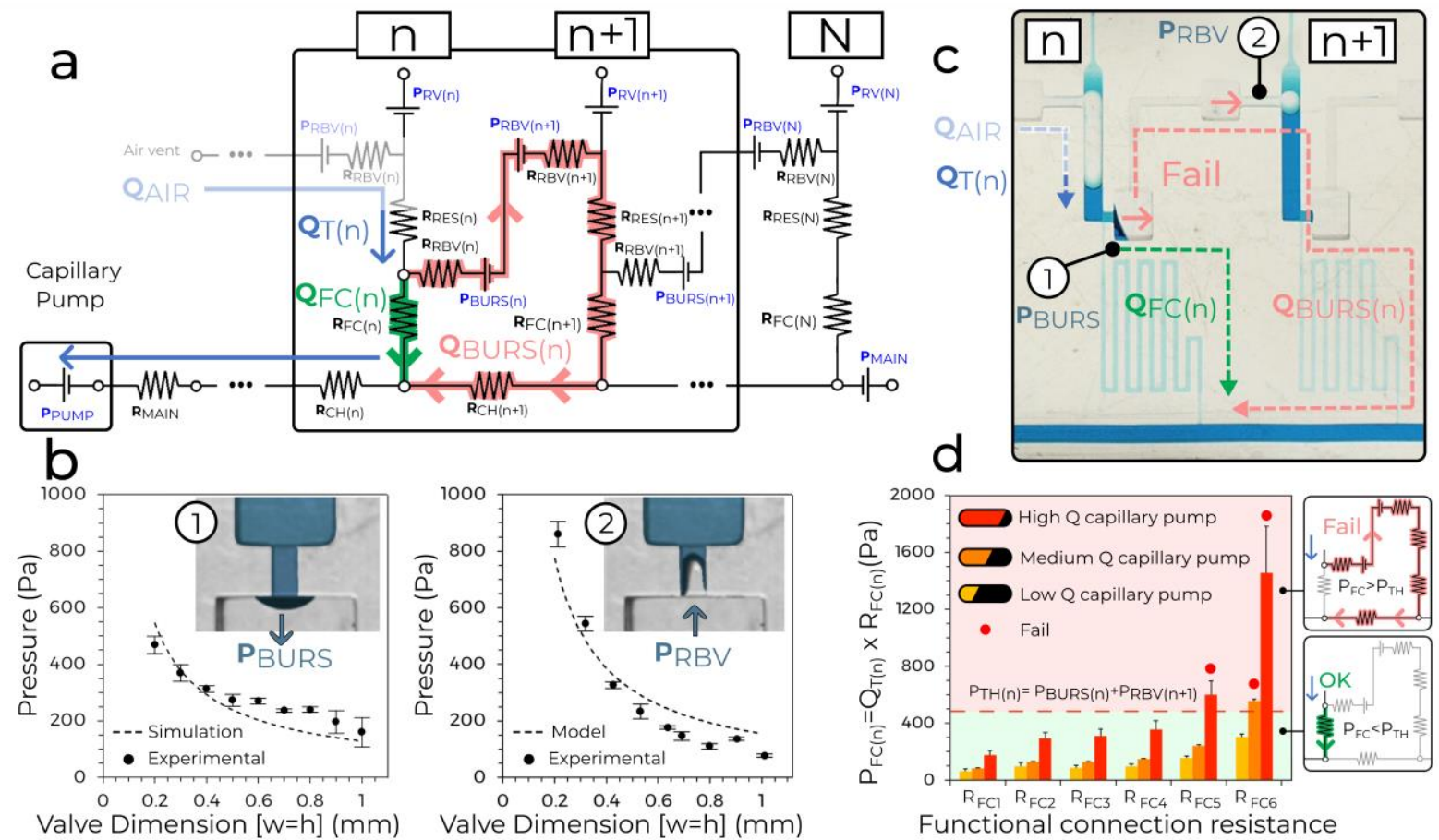

Fig. 2. Circuit analysis and experiments identify operational window for MCRs. (a) The equivalent electrical circuit of the MCR units shown in Fig. 1. (b) The burst pressure (1) and retention pressure (2) for a valve with different dimensions fitted with a numerical and an analytical model, respectively. Error bars are standard deviations from 3 experiments. (c) Illustration of failure for a CDV where liquid leaks inside the air link leading to draining of reservoir $n+1$ prematurely. (d) Testing 6 MCRs with increasing $\mathrm{R}_{\mathrm{FC}}$ and three different paper pumps to determine the effect of varying the flow rate ( $\mathrm{n}=3$ for each paper pump and $R_{F C}$ ). As predicted, the CDVs fail when the pressure drop across the functional connection $P_{F C(n)}$ exceeds the CDV threshold pressure $P_{B U R S(n)}+P_{R B V(n+1)}$. 


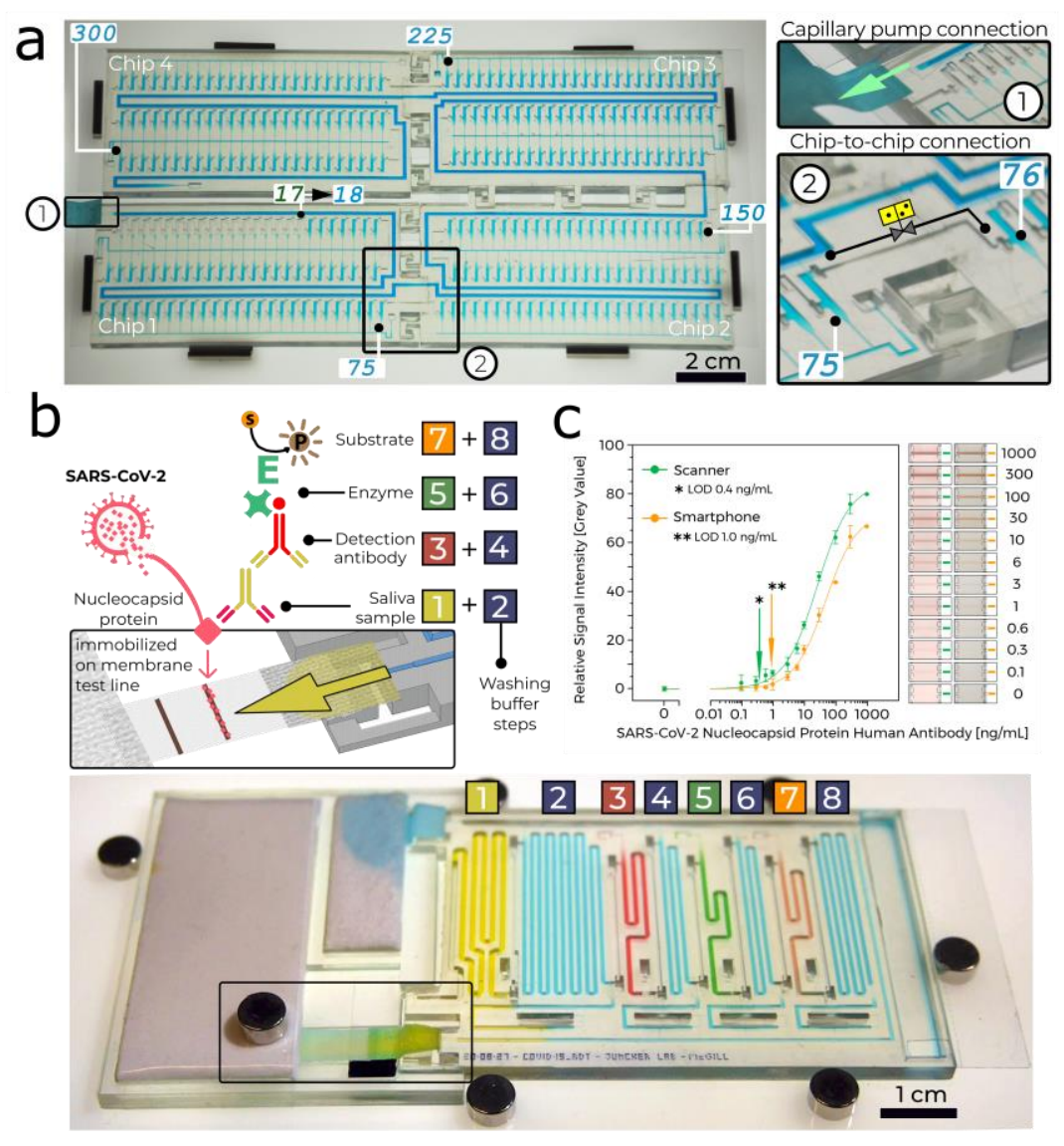

Fig. 3. Large-scale MCR and quantitative COVID-19 serology test. (a) A MCR of 300 aliquots stored in $4.9 \mu 1$ reservoirs across four chained and interconnected chips. See Video S2. (b) Proof-of-concept diagnostic test for SARS-CoV-2 antibodies in saliva comprising two clickable chips, one with paper pumps and membrane, and one with sample, reagents and washing buffers. Sequential, preprogrammed release via MCR is triggered by click-connecting chips and paper pump as detailed in the step-by-step diagram, assay schematic (left), and chip screenshot (bottom), see Video S3. The MCR supplies 4 reagents and 4 buffers in sequence. The functionality includes standard delivery and removal (by flushing) of solutions, individual metering via reservoir size $(40 \mu \mathrm{l}-200 \mu \mathrm{l})$, flow speed and time control via the flow resistance of the functional connection and the capillary-pressure of the paper pump. The enzymatic amplification produces a brown precipitate line visible to the naked eye. (c) Assay binding curve obtained by spiking antibody into saliva, and imaging by scanner and cell phone with representative images of the detection zone for each concentration, indicating the potential for quantitative point-of-care assays. Error bars are standard deviations of 3 replicate measurements 
for concentrations of $0-10 \mathrm{ng} / \mathrm{ml}$, and of 2 replicate measurements for concentrations of $30-$ $300 \mathrm{ng} / \mathrm{ml}$.

We designed a chip-to-chip interface with a leakage-free connection for liquid (main channel) and air (connecting the CDVs), respectively, and connected 4 chips with $75 \mathrm{MCR}$ each, Fig. 3a and Video S2. This result demonstrates integrated, large scale fluidic operations by 'passive' capillary microfluidics, beyond the capability of many active microfluidic systems.

\section{Automated COVID-19 saliva antibody test}

We developed a point-of-care test for detecting antibodies against SARS-CoV-2 in saliva. The MCR drives a sequence of 8 steps commonly used in laboratory enzyme linked immunosorbent assay (ELISA), Fig. 3b. Conventional lateral flow assays with pre-dried reagents cannot implement rinsing steps or enzymatic amplification, and accurate readout is limited to within a short time-window. Here, we used 3,3'-Diaminobenzidine as a substrate which upon enzymatic conversion produced a brown, persisting precipitate that could serve both as an immediate readout and a record for archival. The assay result can be visualized by naked eye, or quantified using a scanner or a smartphone integrated with a simple folded origami box to minimize light interference, with a sensitive, quantitative, and reproducible output. (Fig. 3c and Supplementary Fig. S5). 


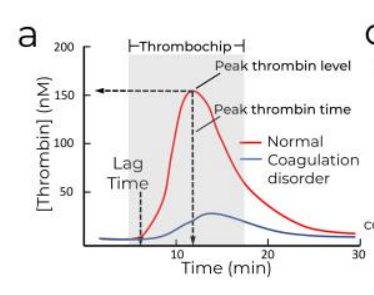

b

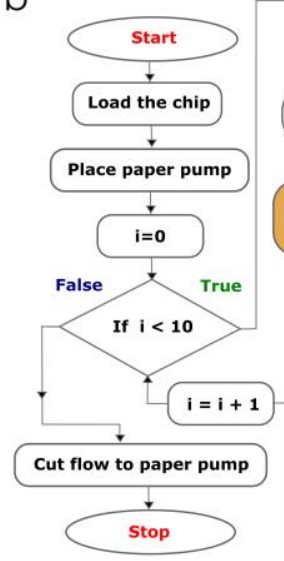

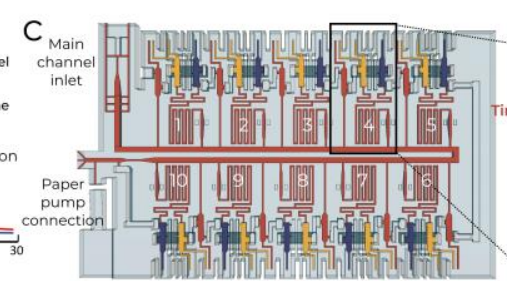

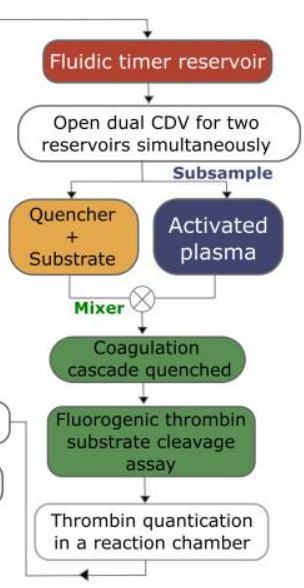

d

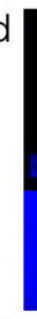

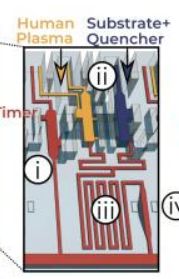

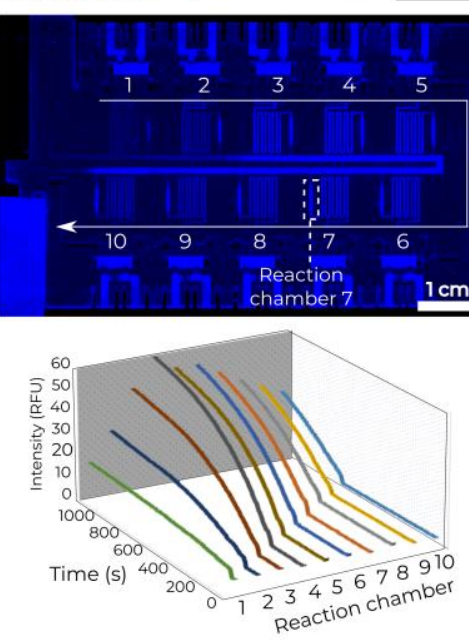

(i) Fluidic (ii) Simultaneous $_{\text {Timelease }}$ Mix (ii) Signal

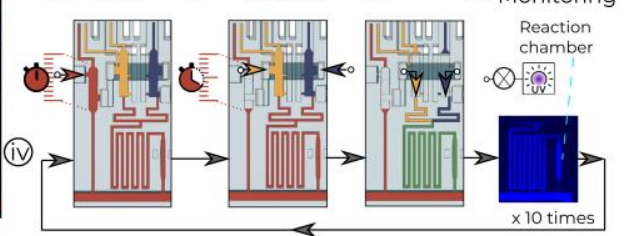

$\mathrm{f}$

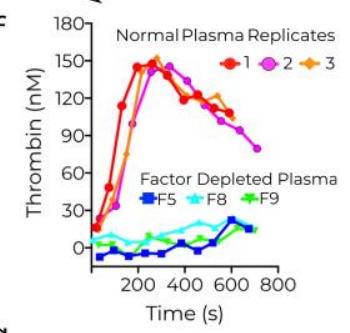

9

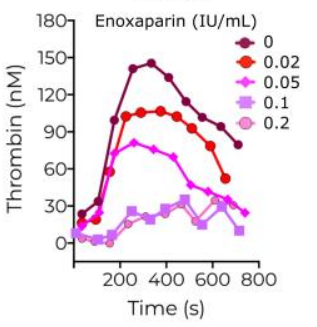

Fig. 4. Automated microfluidic thrombogram. (a) Model thrombograms for normal (red) and hemophilic (blue) plasma. Grey box is the time window of the thrombochip. (b) Thrombogram operations and algorithm encoded in the thrombochip. (c) Schematic of the thrombochip with insets highlighting (i) timer, (ii) simultaneous release of plasma and reagents (quencher and substrate), (iii) mixing, and (iv) flow-stop in the reaction chamber and monitoring of the fluorescence time-course signal. (d) Dark field image of UV illuminated thrombochip in operation revealing fluorescent signal. (e) Fluorescence thrombin quantification for the ten 1 min-interval timepoints during the activation of the coagulation cascade. Abridged thrombograms of human plasma that is (f) normal (3 replicates of pooled plasma), factor deficient (F5, F8, F9), and (g) mixed with anticoagulant drug (Enoxaparin) at different concentrations.

\section{Automated microfluidic thrombogram}

Thrombograms are the gold standard to monitor blood coagulation cascade as they provide the time course of active thrombin concentrations in clotting plasma, reflected in peak height and shape (Fig. 4a). They are used to investigate thrombotic and hemophilic disorders, 
assess thrombotic tendency and determine the effect of antithrombotic drugs. Thrombograms by subsampling are performed by activating plasma, triggering the coagulation cascade and thrombin production, and aliquoting it in wells, followed by addition at $1 \mathrm{~min}$ intervals of a quencher solution (EDTA) immediately stopping the thrombin production, and of a substrate that reacts with the accumulated thrombin, generating a fluorescent product. The procedure is lengthy and laborious, and had not been adapted to microfluidics previously. We devised an algorithm Fig. 4b for automating it, and designed a complex MCR with cascaded, iterative, and branching fluidic operations named thrombochip, Fig. 4c (and Supplementary Fig. S6 and Video S4).

Upon loading and triggering, and without further intervention, the activated plasma and the reagent (quencher and substrate) were released at 1 min intervals from the ten pairs of reservoirs, quickly mixed, and stored in a 2.1- $\mu$ l-reaction chamber with a width of $500 \mu \mathrm{m}$ for fluorescence signal generation and readout using a camera, Videos S5 and S6. Human pooled plasma, plasma with coagulation disorders such as hemophilia A (Factor VIII-deficient), hemophilia B (Factor IX-deficient) and Factor V-deficient were successfully analyzed, as well as the dose-response of an anticoagulant drug was measured, Fig. 4f, g.

\section{Conclusion and Discussion}

MCRs introduce deterministic, modular and programmable chain reactions at the mesoscale and constitute a new concept for autonomous, programmable liquid operations and algorithms via control of both hydraulic and pneumatic flow and connectivity. The automation of complex and repetitive liquid handling operations has so far only been possible with a computer, software programs, and cumbersome peripheral equipment, either robotics, or in the case of microfluidics, ${ }^{6}$ systems to supply reagents, power, or flow control. ${ }^{8-11} \mathrm{MCR}$ introduces mesoscale chain reactions as a frugal, integrated, scalable, and programmable process that power integrated lab-on-a-chips.

The MCR chip micro-architecture is simultaneously the circuit and the code of the chain reaction, is manufacturable with a variety of techniques, and scalable along two distinctive paths: Firstly, following microelectronics example and Moore's law, by shrinking and increasing the number of features per unit area, and per unit volume (e.g. by using 3D printing). Secondly, by expanding the overall size of MCRs by interconnecting and chaining chips, and, inspired by trees that draw liquids over $100 \mathrm{~m}$ in height, linking them to powerful capillary pumps. ${ }^{21} \mathrm{We}$ 
anticipate numbers of steps far beyond the 300 shown here, and much more complex algorithms than the thrombochip.

MCRs are generalizable, compatible with positive pressure operations, and could be interfaced with active microfluidics and robotic liquid handling systems. Spontaneous, capillaryflow MCRs may be further improved too with permanently hydrophilic resins or coatings, liquid storage pouches, and pre-dried reagents ${ }^{22}$, notably for point-of-care applications, and any other uses. An end-user, by simply depositing a drop of solution at the inlet, could trigger a choreography of timed operations including aliquoting, delivery, mixing, flushing and reaction of multiple chemicals. As MCRs can be 3D printed and monolithically encoded in a chip, the entry barrier is very low (entry-level resin-based printers cost $<\$ 300)$. MCRs may be homemanufactured easily, or mail-ordered, opening the way for rapid dissemination, and for new inventions, advances, and for downloadable and printable microfluidic apps.

\section{Data availability}

15 Data not presented in the article or supplementary material will be available upon request.

\section{References}

1. Denisov, E., Sarkisov, O. \& Likhtenshtein, G. I. Chemical Kinetics: Fundamentals and Recent Developments. Elsevier (2003).

2. Award ceremony speech - NobelPrize.org. Nobel Media AB 2021

https://www.nobelprize.org/prizes/chemistry/1956/ceremony-speech/.

3. Mullis, K. et al. Specific enzymatic amplification of DNA in vitro: The polymerase chain reaction. Cold Spring Harb. Symp. Quant. Biol. 51, 263-273 (1986).

4. Saiki, R. et al. Primer-directed enzymatic amplification of DNA with a thermostable DNA polymerase. Science (80-. ). 239, 487-491 (1988).

5. Dirks, R. M. \& Pierce, N. A. Triggered amplification by hybridization chain reaction. Proc. Natl. Acad. Sci. U. S. A. 101, 15275-15278 (2004).

6. Sackmann, E. K., Fulton, A. L. \& Beebe, D. J. The present and future role of microfluidics in biomedical research. Nature 507, 181-189 (2014).

7. Manz, A., Graber, N. \& Widmer, H. M. Miniaturized total chemical analysis systems: A novel concept for chemical sensing. Sensors Actuators B. Chem. 1, 244-248 (1990).

8. Thorsen, T., Maerkl, S. J. \& Quake, S. R. Microfluidic Large-Scale Integration. Science (80-. ). 298, 580-584 (2002).

9. Choi, K., Ng, A. H. C. C., Fobel, R. \& Wheeler, A. R. Digital microfluidics. Annu. Rev. Anal. Chem. 5, 413-40 (2012).

10. Strohmeier, O. et al. Centrifugal microfluidic platforms: advanced unit operations and applications. Chem. Soc. Rev. 44, 6187-6229 (2015). 
11. Easley, C. J. et al. A fully integrated microfluidic genetic analysis system with sample-inanswer-out capability. Proc. Natl. Acad. Sci. U. S. A. 103, 19272-7 (2006).

12. Ozer, T., McMahon, C. \& Henry, C. S. Advances in Paper-Based Analytical Devices. Annu. Rev. Anal. Chem. 13, 85-109 (2020).

13. Olanrewaju, A., Beaugrand, M., Yafia, M. \& Juncker, D. Capillary microfluidics in microchannels: From microfluidic networks to capillaric circuits. Lab on a Chip vol. 18 2323-2347 (2018).

14. Safavieh, R. \& Juncker, D. Capillarics: Pre-programmed, self-powered microfluidic circuits built from capillary elements. Lab Chip 13, 4180-4189 (2013).

15. Wang, X., Hagen, J. A. \& Papautsky, I. Paper pump for passive and programmable transport. Biomicrofluidics 7, 014107 (2013).

16. Kokalj, T., Park, Y., Vencelj, M., Jenko, M. \& Lee, L. P. Self-powered imbibing microfluidic pump by liquid encapsulation: SIMPLE. Lab Chip 14, 4329-4333 (2014).

17. Dal Dosso, F. et al. Creasensor: SIMPLE technology for creatinine detection in plasma. Anal. Chim. Acta 1000, 191-198 (2018).

18. Olanrewaju, A. O., Robillard, A., Dagher, M. \& Juncker, D. Autonomous microfluidic capillaric circuits replicated from 3D-printed molds. Lab Chip 16, 3804-3814 (2016).

19. Achille, C. et al. 3D Printing of Monolithic Capillarity-Driven Microfluidic Devices for Diagnostics. Adv. Mater. 2008712 (2021) doi:10.1002/adma.202008712.

20. Olanrewaju, A. O., Robillard, A., Dagher, M. \& Juncker, D. Autonomous microfluidic capillaric circuits replicated from 3D-printed molds. Lab Chip 16, 3804-3814 (2016).

21. Wheeler, T. D. \& Stroock, A. D. The transpiration of water at negative pressures in a synthetic tree. Nature 455, 208-212 (2008).

22. Gökçe, O., Castonguay, S., Temiz, Y., Gervais, T. \& Delamarche, E. Self-coalescing flows in microfluidics for pulse-shaped delivery of reagents. Nature 574, 228-232 (2019).

Acknowledgments: We acknowledge the help from Justin-Lessard Wajcer, Jay Pimprikar and Marzieh Miyabargheri, Yannis Pascalidis as well as Thomas Gervais for insightful comments.

Funding: This work was supported by NSERC Strategic Project Grant STPGP 494495-16 and, NSERC Alliance Grant ALLRP 551058-20 and an McGill MI4 SCRF grant. M.Y. acknowledges FRQNT postdoctoral fellowship \#260284. A.S.K. acknowledges FRQNT postdoctoral fellowship (\#267919). D.J. acknowledges support from a Canada Research Chair in Bioengineering;

Author contributions: Methodology, Data curation, and Formal Analysis: D.J. A.O. and M.Y. designed and tested the two-layered MCR. M.Y. designed and tested the monolithic MCR. O.Y., A.P., J.R. and Z.J., A.S. and A.P. designed MCR CC for experiments and analyzed the data for the COVID-19 antibody test. O.Y. performed the pressure characterization tests. M.Y. and A.N. performed the experiments and analyzed the data for the thrombochip. M.Y. designed and performed the experiments for the CDV failure. A.P. and A.S.K. performed the experiments for the 300 MCRs. M.Y. performed the CT scan and dimensions characterization. A.S. and A.P. performed the contact angle measurements. G.K. performed the simulation for the burst pressure on COMSOL. D.J. guided design and analysis of all experiments. Visualization: M.Y, O.Y, A.N. and D.J. prepared the main figures. M.Y. and Z.J worked on the 3D visualizations. M.Y., O.Y., A.O., A.P., A.S.K, Z.J. and A.N. captured all the images and videos. G.K. and M.Y. 
worked on editing all the videos. Writing - original draft: M.Y., A.O., A.N. and D.J. Writing review \& editing: M.Y., A.O., A.P., Z.J, J.R., G.K. and D.J. Project conception, administration, and supervision A.N. and D.J.

Competing interests: M.Y., A.O. and D.J. are inventors on a ROI submitted to McGill and licensed. All other Authors declare no competing interests.

\section{Additional information:}

Supplementary Information is available for this paper.

Correspondence and requests for materials should be addressed to:

David Juncker

6500-740 Dr Penfield Avenue

Montreal, QC. CA. H3A 0G1

david.juncker@mcgill.ca

Phone: +1 (514) 398 7676, Fax: +1 (514) 3981790 


\section{Methods:}

Chip design and fabrication

The chips were designed using AutoCAD (Autodesk) and exported as .STL files for 3D printing. CCs encoding MCRs were made with a digital micromirror display (DMD) 3D printer (Miicraft 100, Creative Cadworks, Toronto, Canada) using a transparent resin (Rapid Model Resin Clear, Monocure 3D, Regents Park, NSW, Australia) purchased from filaments.ca (Mississauga, Canada). The following printing parameters were used: The layer thickness was 20 $\mu \mathrm{m}$, the exposure time $1.5 \mathrm{~s}$ per layer, while the exposure time for the base layer was $10 \mathrm{~s}$ with 4 transition buffer layers. Following completion of the print, the chips were cleaned with isopropanol and post-cured for 1 min under UV (Professional CureZone, Creative Cadworks, Toronto, Canada)

Microchannels with cross-sections ranging from $250 \times 100 \mu \mathrm{m}^{2}$ to $1500 \times 1000 \mu \mathrm{m}^{2}$ were fabricated and hydrophilized by plasma activation for $10 \mathrm{~s}$ at $30 \%$ power (PE50 plasma chamber, Plasma Etch, Carson City, USA).

CCs were sealed with a delayed tack adhesive (9795R microfluidic tape, 3M) forming the cover. Whatman filter papers are used as paper pumps to run the chips (Whatman filter paper grade 4, 1 and 50 Hardened, Cytiva, Marlborough, USA). Absorbent pads were clamped on the filter for chips running the COVID assay (Electrophoresis and Blotting Paper, Grade 238, Ahlstrom-Munksjo Chromatography, Helsinki, Finland).

\section{Chip-to-chip connections}

To obtain a leakage-free connection, a thin layer of an uncured photoresin, prepared by mixing poly (ethylene glycol) diacrylate (PEG-DA MW 258, Sigma Aldrich) and Irgacure-819 $(1 \% \mathrm{w} / \mathrm{w})$, and applied to all of the chip-to-chip interfaces. Next, the chips were assembled and exposed to UV light for in a UV chamber (320-390 nm, UVitron Intelliray 600 at 50\% intensity) for $30 \mathrm{~s}$ to cure the resin and seal the connections.

\section{Videos and image processing}

Videos and images were recorded using a Panasonic Lumix DMC-GH3K. Structural images of the chip and the embedded conduits were obtained using a micro CT (Skyscan 1172, Bruker, Billerica, USA) and used to confirm the dimensions. Contact angles were measured based on side view images $(n=3)$ and analyzed using the Dropsnake extension in Image J.

$35 \quad$ Modeling and Calculations

The theoretical burst pressures of capillary stop valves were calculated by solving the flow field using the finite element method (FEM) with COMSOL Multiphysics 5.5 (Burlington, MA). Experimentally measured contact angles $\left(100^{\circ}\right.$ and $40^{\circ}$ for the cover and the channel, respectively) were used to solve two-phase capillary flow using the level-set method. The capillary flows leading up to the stop valve was solved for a time period of 0-0.02 seconds with a time step of $1 \times 10^{-5} \mathrm{~s}$. The inlet pressure was varied with $10 \mathrm{~Pa}$ increment for each simulation, until burst was observed based on continuous flow.

COVID-19 serological test

SARS-CoV-2 nucleocapsid protein was purchased from Sino Biological, Inc., Beijing, China (Catalog no 40588-V08B). Human Chimeric antibody against SARS-CoV-2 nucleocapsid 
protein was purchased from Genscript Biotech, Piscataway, USA (A02039). SIGMAFASTTM 3,3'-Diaminobenzidine (DAB) tablets were purchased from Sigma-Aldrich, Oakville, Canada. Biotinylated Goat-anti-Human antibody was purchased from Cedarlane, Burlington, Canada (GTXHU-003-DBIO). Pierce ${ }^{\text {TM }}$ Streptavidin Poly-HRP (21140) was purchased from ThermoFisher, Ottawa, Canada.

Nitrocellulose membranes (Whatman FF80HP Plus nitrocellulose-backed membranes, Cytiva, USA) were designed and cut into $5.2 \mathrm{~mm}$ wide strips using the Silhouette Portrait paper cutter (Silhouette, Lindon, USA). Membranes were striped with a $5 \mathrm{~mm}$ wide test line of 0.25 $\mathrm{mg} / \mathrm{mL}$ SARS-CoV-2 nucleocapsid protein delivered using a programmable inkjet spotter (sciFLEXARRAYER SX, Scienion, Berlin, Germany). The test line consists of 4 lanes of 50 droplets of about $350 \mathrm{pL}$ printed $100 \mu \mathrm{m}$ apart from each other. 8 passes of 25 droplets were used for each lane on even and odd positions to allow solution absorption in between passes. The membranes were then dried for $1 \mathrm{~h}$ at $37{ }^{\circ} \mathrm{C}$ before blocking by dipping into $1 \%$ BSA in $1 \mathrm{X}$ PBS solution until completely wet, then retrieved and left to dry for $1 \mathrm{~h}$ at $37^{\circ} \mathrm{C}$ and then stored with desiccant at $4{ }^{\circ} \mathrm{C}$ until use the next day.

For the antibody assays, nitrocellulose strips were connected to glass fiber conjugate pad strips (G041 SureWick, Millipore Sigma, Oakville, Canada) and clamped onto the CC outlet. Human saliva was extracted with oral swabs (SalivaBio, Salimetrics, State College, USA), followed by centrifugation, $10 \mathrm{X}$ dilution and filtration with 0.22 -micron filters. Human Chimeric antibody against SARS-CoV-2 nucleocapsid protein at 0 to $1000 \mathrm{ng} / \mathrm{ml}$ was spiked into diluted saliva and loaded to the sample reservoirs.

Image analysis on the nitrocellulose strips

The nitrocellulose strips were removed from CC modules and left to dry for one hour. The dry strips were then scanned at $600 \mathrm{dpi}$ and grey values of test lines were extracted with ImageJ software. Local background grey values were taken at 30 pixels above each test line and subtracted from test line values. The standard curve was then generated by subtracting negative control signal value from all data points. Smartphone imaging of the nitrocellulose strips was done with a Huawei P10 smartphone with a 12-megapixel image sensor and a rear camera with a 27-mm focal length (Huawei, Shenzhen, China). A customized box was cut and folded with black cardboard paper to block ambient light. The box had two slots fitting the size of camera and nitrocellulose strip, respectively, to ensure accurate alignment of strip for readout. Images were taken with on-camera dual tone LED flash on at full power. Analysis of smartphone-taken images was done as described above.

Automated microfluidic thrombogram (Thrombochip)

Citrated human plasma, fluorogenic thrombin substrate Z-GGR-AMC and Enoxaparin were purchased from Sigma-Aldrich, Oakville, Canada; Batroxobin was from Prospec, East Brunswick, USA; Technothrombin TGA RC High reagent was from Diapharma, West Chester, USA; Human thrombin, Factor V (FV)-, Factor VIII (FVIII)- and Factor IX (FIX)-deficient plasma were from Haematologic Technologies, Essex Junction, USA; (4-(2-hydroxyethyl)-1piperazineethanesulfonic acid) (HEPES), Ethylenediaminetetraacetic acid (EDTA), $\mathrm{CaCl}_{2}$ were from Sigma-Aldrich, Oakville, Canada.

Human plasma (pooled normal or factor-deficient) were defibrinated by the addition of batroxobin (final concentration $0.6 \mathrm{BU} / \mathrm{mL}$ ). The mixtures were incubated at room temperature 
for $20 \mathrm{~min}$, followed by an additional incubation at $4{ }^{\circ} \mathrm{C}$ for $1 \mathrm{~h}$. The mixtures were then centrifuged at 10,000-g for $10 \mathrm{~min}$ to remove the fibrin clot and other debris. Defibrinated plasma were collected from the supernatant.

A solution containing $21 \%$ defibrinated plasma, $48 \%$ Technothrombin TGA RC High reagent (high phospholipid and relipidated tissue factor content) and $20 \mathrm{mM} \mathrm{CaCl}_{2}$ in $25 \mathrm{mM}$ HEPES at $\mathrm{pH} 7.4$ was loaded into the sample reservoirs of the thrombochip. A substrate solution containing $420 \mu \mathrm{M}$ Z-GGR-AMC, $30 \mathrm{mM}$ EDTA in $25 \mathrm{mM}$ HEPES at $\mathrm{pH} 7.4$ was loaded into the reagent reservoirs. All solutions were equilibrated to room temperature for $20 \mathrm{~min}$ before loading. Coagulation-inhibited plasma contained Enoxaparin at final concentrations of 0 to 1.0 anti-Xa units $/ \mathrm{mL}$ or IU/mL. The samples and reagents were loaded on the chip after initiating the coagulation cascade. The paper pump was connected to the chip to start the flow after 5 min from initiating the coagulation cascade. Fluorescence signals generated in the reaction chambers were monitored by illuminating the thrombochip with UV light at $365 \mathrm{~nm}$ with $20 \mathrm{~W}$ (realUV ${ }^{\mathrm{TM}}$ LED Flood Light, Waveform Lighting, USA) and the visible $440 \mathrm{~nm}$ fluorescence emission signals measured by imaging at $5 \mathrm{~s}$ intervals using a Panasonic Lumix DMC-GH3K digital camera (f/3.5, Exposure time: $2 \mathrm{~s}$, ISO-200). The rate of fluorescence signal generation in each reaction chamber (i.e. the slope of the recorded fluorescence generation curve) equals the rate of substrate turnover by thrombin, which was used to deduce the amount of thrombin generated using a standard curve. Image J was used to analyze the images for fluorescence intensity,

$\underline{\text { Standard curve for thrombin quantification }}$

10 human thrombin solutions at concentrations ranging from 0 to $300 \mathrm{nM}$ in $25 \mathrm{mM}$ HEPES at $\mathrm{pH} 7.4$ were loaded into the 10 sample reservoirs in the thrombochip. A substrate solution containing $420 \mu \mathrm{M}$ Z-GGR-AMC, $30 \mathrm{mM}$ EDTA in $25 \mathrm{mM}$ HEPES at pH 7.4 was loaded into the reagent reservoirs. The standard curve was constructed by plotting the slope of the recorded fluorescence generation curve in each reaction chamber against the known thrombin concentration of the solution that was loaded to the corresponding sample reservoir. 